October 2001

PAR-LPTHE 01/?

\title{
Universality of coupled Potts models
}

\author{
Vladimir S. Dotsenko (1), Jesper Lykke Jacobsen (2), \\ Xuan Son Nguyen (1), and Raoul Santachiara (1) \\ (1) LPTHE冈, Université Pierre et Marie Curie, Paris VI \\ Université Denis Diderot, Paris VII \\ Boîte 126, Tour 16, $1^{\text {er }}$ étage \\ 4 place Jussieu, F-75252 Paris Cedex 05, France. \\ (2) Laboratoire de Physique Théorique et Modèles Statistiques, \\ Université Paris-Sud, Bâtiment 100, F-91405 Orsay, France.
}

\begin{abstract}
We study systems of $M$ Potts models coupled by their local energy density. Each model is taken to have a distinct number of states, and the permutational symmetry $S_{M}$ present in the case of identical coupled models is thus broken initially. The duality transformations within the space of $2^{M}-1$ multi-energy couplings are shown to have a particularly simple form. The selfdual manifold has dimension $D_{M}=2^{M-1}-1$. Specialising to the case $M=3$, we identify a unique nontrivial critical point in the three-dimensional selfdual space. We compare its critical exponents as computed from the perturbative renormalisation group with numerical transfer matrix results. Our main objective is to provide evidence that at the critical point of three different coupled models the symmetry $S_{3}$ is restored.
\end{abstract}

*Unité Mixte de Recherche CNRS UMR 7589. 


\section{Introduction}

In the study of coupled models, and also of disordered models in their replica formulation, the permutation group symmetry $S_{M}$ is supposed to play an essential role [1, 2, 3]. Namely, the interaction part of the action for a set of $M$ identical coupled models

$$
A_{\text {int }} \propto \int \mathrm{d}^{2} x g \sum_{a \neq b} \varepsilon_{a}(x) \varepsilon_{b}(x)
$$

is explicitly invariant with respect to any permutation of the models. Here $a$ and $b$ are replica indices, and $\left\{\varepsilon_{a}(x)\right\}$ designates the set of local energy operators. In the lattice definition of such coupled models the interaction part of the Hamiltonian takes a similar form, with only $\int \mathrm{d}^{2} x$ replaced by a summation and $\left\{\varepsilon_{a}(x)\right\}$ by an appropriate lattice expression'.

When one introduces asymmetric couplings, by generalising the common coupling constant $g$ to a matrix $g_{a b}$,

$$
A_{\text {int }} \propto \int \mathrm{d}^{2} x \sum_{a \neq b} g_{a b} \varepsilon_{a}(x) \varepsilon_{b}(x)
$$

a perturbative renormalisation group $(\mathrm{RG})$ analysis reveals that the $S_{M}$ symmetry is restored at the fixed point. A detailed study of this scenario, within the $\epsilon$-type perturbative RG for coupled Potts models, was carried out in [4]. Supposing all components of $g_{a b}$ to stay of order $\epsilon$, their initial values being all positive], it was shown in [4] that the only non-trivial fixed point, having one attractive direction, all other directions being repulsive, is that with $g_{a b} \equiv g$. Th This type of restoration of the symmetry $S_{M}$ could be

\footnotetext{
${ }^{1}$ In the case of the Potts model with nearest-neighbour spins $\sigma_{i}^{(a)}$ and $\sigma_{j}^{(a)}$ in the replica $a$, one has $\varepsilon_{a}(x) \sim 1-\delta\left(\sigma_{i}^{(a)}, \sigma_{j}^{(b)}\right)$.

${ }^{2}$ In [4] the interaction part of the action $A_{\text {int }}$ (1.2) was actually defined with an extra minus sign, so that initially all the components of $g_{a b}$ were taken to be negative.

${ }^{3}$ Ref. [4] also identified other fixed points, which are related to the basic one (with all $g_{a b}$ equal and of the same sign) by changing the sign of some of its components. This is equivalent to switching the sign of a certain number of energy operators. This last operation is a symmetry of the individual Potts models, corresponding to their self-duality (note however such duality transformations on the individual models are not directly related to the global duality transformations on the entire coupled system to be discussed in section 3). This argument implies that the critical properties of the various critical points classified in [4] should be equivalent.
} 
called "soft universality".

In this paper we are going to argue for a "strong universality" in the criticality of coupled models. We shall mainly be interested in coupling $M=3$ different models (1.2), with $\left\{\varepsilon_{a}\right\}$ belonging to Potts models with different number of states $\left\{q_{1}, q_{2}, q_{3}\right\}$. This breaks the permutational symmetry in a "strong" sense. Still, the RG calculations show the existence of a single fixed point with all $\left\{g_{a b}\right\}$ being positive, like it is the case for identical models.

To our knowledge, Simon [5] was the first to apply the RG analysis to a set of different coupled Potts models. The most general model studied by this author was that of $M_{1}$ Potts models with $q_{1}$ states and $M_{2}$ Potts models with $q_{2}$ states $\left(q_{1} \neq q_{2}\right)$, all of them being coupled. After determining the fixed point structure, he computed the dimensions of the spin operators, as well as the RG equations for the energy operators to two-loop order. The effect of disorder on these coupled systems was also analysed.

Here we generalise the RG calculations of [5] to the case of three different coupled Potts models $\left(q_{1} \neq q_{2} \neq q_{3}\right)$. We shall compute the dimensions of energy operators, with a special focus on the symmetry of the theory, at the non-trivial fixed point that generalises the one found in [2] for three identical models. Within the space of couplings $\left\{g_{a b}\right\}$, the new fixed point is stable in one direction and unstable in the others, the topology of the RG flows being similar to those of [2]. But there is also one apparent difference: The permutational symmetry has disappeared, the coupled models being different.

The purpose of this paper will be to provide evidence, by using various methods, that at the fixed point of three different coupled models the apparently lost symmetry $S_{3}$ is restored, implying a "strong universality".

This symmetry restoration cannot be observed on the level of the initial action (1.2), as discussed above, nor is it visible in the perturbative RG treatment, or in the Hamiltonian of the explicit lattice realisation. This is because $\left\{\varepsilon_{a}\right\}$ are the energy operators of different models, with different scaling dimensions in particular.

\footnotetext{
${ }^{4}$ The duality transformations of section 3 are however valid for general $M$.
} 
The way we shall check for the restoration of the symmetry is by looking at the spectrum of scaling dimensions at the new fixed point, within the sector of energy operators. Like in the case of identical models [1, 2, 3], the RG analysis implies that the three energy operators of the decoupled models $\left\{\varepsilon_{1}(x), \varepsilon_{2}(x), \varepsilon_{3}(x)\right\}$ will rearrange as three particular linear combinations so as to form the new primary operators at the fixed point of the coupled models.

In the case of identical models the corresponding linear combinations are easy to guess on symmetry grounds. The irreducible representations (irreps) of the group $S_{3}$ in the basis $\left\{\varepsilon_{1}(x), \varepsilon_{2}(x), \varepsilon_{3}(x)\right\}$ consist of a (symmetric) singlet

$$
\varepsilon_{\mathrm{S}}(x)=\varepsilon_{1}(x)+\varepsilon_{2}(x)+\varepsilon_{3}(x)
$$

and an (antisymmetric) doublet

$$
\left\{\begin{array}{l}
\varepsilon_{\mathrm{A}_{1}}=\varepsilon_{1}(x)-\varepsilon_{2}(x) \\
\varepsilon_{\mathrm{A}_{2}}=\varepsilon_{1}(x)-\varepsilon_{3}(x)
\end{array}\right.
$$

that act as the new primary operators at the fixed point [1, 2, 3]. The fact that the operators $\varepsilon_{\mathrm{A}_{1}}$ and $\varepsilon_{\mathrm{A}_{2}}$ belong to the same two-dimensional irrep means that their dimensions must coincide: $\Delta\left(\varepsilon_{\mathrm{A}_{1}}\right)=\Delta\left(\varepsilon_{\mathrm{A}_{2}}\right)$. These are however in general different from the dimension $\Delta\left(\varepsilon_{\mathrm{S}}\right)$ of the one-dimensional irrep.

When coupling different models, the corresponding linear combinations of $\varepsilon_{1}, \varepsilon_{2}, \varepsilon_{3}$ will have more complicated coefficients which have to be calculated by the RG technique. One will find something of the form:

$$
\left\{\begin{array}{l}
\varepsilon_{1}^{*}=K_{11} \varepsilon_{1}+K_{12} \varepsilon_{2}+K_{13} \varepsilon_{3} \\
\varepsilon_{2}^{*}=K_{21} \varepsilon_{1}+K_{22} \varepsilon_{2}+K_{23} \varepsilon_{3} \\
\varepsilon_{3}^{*}=K_{31} \varepsilon_{1}+K_{32} \varepsilon_{2}+K_{33} \varepsilon_{3}
\end{array}\right.
$$

Since the initial dimensions $\Delta\left(\varepsilon_{1}\right), \Delta\left(\varepsilon_{2}\right), \Delta\left(\varepsilon_{3}\right)$ of the decoupled models differ, one may expect that the critical dimensions ( $R G$ eigenvalues) of the newly formed primary operators $\varepsilon_{1}^{*}, \varepsilon_{2}^{*}, \varepsilon_{3}^{*}$ might all be different.

Our argument is that, in the case of coupling different models, it is not the linear combinations (1.5) which have to be examined to analyse the symmetry, but rather the 
spectrum of their critical dimensions. Permuting $\varepsilon_{1}, \varepsilon_{2}, \varepsilon_{3}$ in the combinations (1.5) does not make much sense because they are different. To permute $\varepsilon_{1}^{*}, \varepsilon_{2}^{*}, \varepsilon_{3}^{*}$, one first has to know their properties, their scaling dimensions, to decide if it makes sense or not.

The conclusion will be that one has to study the spectrum of dimensions at the new fixed point. This provides a representation independent information, independent of the way one has defined the theory initially, as by its action (Hamiltonian) in Eq. (1.2).

If the symmetry $S_{3}$ is restored then the dimensions

$$
\Delta\left(\varepsilon_{1}^{*}\right), \Delta\left(\varepsilon_{2}^{*}\right), \Delta\left(\varepsilon_{3}^{*}\right)
$$

should form a singlet and a doublet, as is the case when one couples initially identical models.

The rest of the paper is organised as follows.

In section 2 we analyse the case $M=3$ by perturbative $\mathrm{RG}$ calculations. As will be explained towards the end of that section, in this case the RG results alone are not sufficient to decide whether the $S_{3}$ symmetry is restored or not. We therefore propose to study the coupled models through a particular lattice realisation, following [2]. To that end, in section 3, we extend the duality transformations derived in [6] for symmetrically coupled models to the asymmetric case. The resulting relations are valid for any number $M$ of coupled models, and for the most general asymmetric multi-energy couplings. In particular we determine the selfdual solutions, thus simplifying dramatically the subsequent numerical simulations.

After recalling briefly, in section 4 , the most efficient transfer matrix algorithm constructed in [2], we show how it may be generalised to the case of asymmetrically coupled models. We then turn to the numerical analysis in section 5. We locate the non-trivial critical point on the selfdual manifold for various systems of $M=3$ coupled models, and we provide accurate values of the central charge and the energetic scaling dimensions. We shall find strong evidence for a singlet/doublet spectrum at the new fixed point. This we interpret as a signal of the restoration of the $S_{3}$ symmetry.

Finally, section 6 is devoted to remarks and conclusions. 


\section{Renormalization group analysis}

In the continuum limit, the three coupled Potts models can be represented by the action

$$
\begin{aligned}
A & =\sum_{a=1}^{3} A_{0}^{(a)}+A_{\text {int }} \\
A_{\text {int }} & =\int \mathrm{d}^{2} x \sum_{a \neq b}^{3} g_{a b}^{0} \varepsilon_{a}(x) \varepsilon_{b}(x)
\end{aligned}
$$

Here $\left\{A_{0}^{(a)}\right\}$ represents three decoupled models, or more precisely the three decoupled conformal field theories for these models at their respective critical points. The interaction term $A_{\text {int }}$ makes them coupled. In general, the initial couplings $\left\{g_{a b}^{0}\right\}$ are taken to be different. Since $\left\{\varepsilon_{a}\right\}$ have different dimensions when the models are different, even if we start from identical couplings $g_{a b}^{0}=g_{0}$, they will become different in the course of renormalisation.

We shall parametrise the dimensions of the energy operators $\left\{\varepsilon_{a}\right\}$ as in the papers [2, 4, 5, 7]:

$$
\left(\Delta_{\varepsilon}^{(a)}\right)_{\mathrm{phys}}=1-\frac{3}{2} \epsilon_{a}
$$

where the physical dimension $\left(\Delta_{\varepsilon}^{(a)}\right)_{\text {phys }}$ corresponds to twice the conformal dimension, as usual. The quantity $\epsilon_{a}$, which measures the deviation from the Ising model, appears also in the Coulomb gas parameter

$$
\left(\alpha_{+}^{(a)}\right)^{2}=\frac{4}{3}-\epsilon_{a}
$$

For the Ising model one has $\epsilon=0$, whence $\alpha_{+}^{2}=\frac{4}{3}$ and $\left(\Delta_{\varepsilon}\right)_{\text {phys }}=1$. The parameter $\alpha_{+}^{2}$ is useful in analytic calculations because it is simpler to use than the central charge of the corresponding conformal theory.

The details of the RG calculations are the same as in Refs. [5, 7]. In particular, all the necessary integrals have been calculated in these papers. The generalisation to the case of three different models is straightforward, and we shall therefore only give the final results.

It turns out to be convenient to redefine the expansion parameters $\left\{\epsilon_{a}\right\}$ and the 
coupling constants $\left\{g_{a b}\right\}$ as follows:

$$
\frac{3}{2} \epsilon_{a}=\tilde{\epsilon}_{a} \quad g_{a b}=\frac{1}{4 \pi} \tilde{g}_{a b}
$$

From now on we shall adopt this convention, omitting the tildes throughout for simplicity of notation.

The $\beta$-functions for the interaction (2.2) are found in the form (to two-loop order):

$$
\left\{\begin{array}{l}
\frac{\mathrm{d} g_{12}}{\mathrm{~d} \xi} \equiv \beta_{12}\left(\left\{g_{a b}\right\}\right)=\epsilon_{12} g_{12}-g_{13} g_{23}-\frac{1}{2} g_{12}\left(g_{13}^{2}+g_{23}^{2}\right) \\
\frac{\mathrm{d} g_{13}}{\mathrm{~d} \xi} \equiv \beta_{13}\left(\left\{g_{a b}\right\}\right)=\epsilon_{13} g_{13}-g_{12} g_{32}-\frac{1}{2} g_{13}\left(g_{12}^{2}+g_{32}^{2}\right) \\
\frac{\mathrm{d} g_{23}}{\mathrm{~d} \xi} \equiv \beta_{23}\left(\left\{g_{a b}\right\}\right)=\epsilon_{23} g_{23}-g_{21} g_{31}-\frac{1}{2} g_{23}\left(g_{12}^{2}+g_{31}^{2}\right)
\end{array}\right.
$$

Here $\xi$ is the RG parameter, $g_{a b}=g_{b a}$ by symmetry of the action (2.2), and we have defined $\epsilon_{a b} \equiv \epsilon_{a}+\epsilon_{b}$.

The renormalisation of the energy operators, to second order, is found to be given by the equations

$$
\frac{d \varepsilon_{a}}{d \xi}=-\left(1-\epsilon_{a}\right) \varepsilon_{a}-\sum_{b \neq a} g_{a b} \varepsilon_{b}-\frac{1}{2}\left(\sum_{d \neq a}\left(g_{a d}\right)^{2}\right) \varepsilon_{a}
$$

In matrix form this reads

$$
\begin{aligned}
\frac{\mathrm{d} \varepsilon_{a}}{\mathrm{~d} \xi} & =-\sum_{b=1}^{3} \Delta_{a b} \varepsilon_{b} \\
\Delta_{a b} & =\left(1-\epsilon_{a}\right) \delta_{a b}-\gamma_{a b}\left(\left\{g_{c d}\right\}\right) \\
\gamma_{a b} & =\left(\begin{array}{ccc}
-\frac{1}{2}\left(g_{12}^{2}+g_{13}^{2}\right) & -g_{12} & -g_{13} \\
-g_{12} & -\frac{1}{2}\left(g_{21}^{2}+g_{23}^{2}\right) & -g_{23} \\
-g_{13} & -g_{23} & -\frac{1}{2}\left(g_{31}^{2}+g_{32}^{2}\right)
\end{array}\right)
\end{aligned}
$$

To define the new primary operators and their scaling dimensions at the new fixed point we shall have to diagonalise the matrix $\Delta_{a b}$. It is however more convenient to regroup the terms $-\epsilon_{a} \delta_{a b}-\gamma_{a b}$ so that

$$
\begin{aligned}
\Delta_{a b} & =\delta_{a b}-\Lambda_{a b} \\
\Lambda_{a b} & =\left(\begin{array}{ccc}
\epsilon_{1}-\frac{1}{2}\left(g_{12}^{2}+g_{13}^{2}\right) & -g_{12} & -g_{13} \\
-g_{12} & \epsilon_{2}-\frac{1}{2}\left(g_{21}^{2}+g_{23}^{2}\right) & -g_{23} \\
-g_{13} & -g_{23} & \epsilon_{3}-\frac{1}{2}\left(g_{31}^{2}+g_{32}^{2}\right)
\end{array}\right)
\end{aligned}
$$


and we need only diagonalise the matrix $\Lambda_{a b}$.

The non-trivial zeros of the $\beta$-functions (2.6) are found as

$$
\left\{\begin{array}{l}
g_{12}^{*}=\sqrt{\epsilon_{13} \epsilon_{23}}\left\{1-\frac{1}{2} \epsilon_{12}-\frac{1}{4}\left(\epsilon_{13}+\epsilon_{23}\right)\right\} \\
g_{13}^{*}=\sqrt{\epsilon_{12} \epsilon_{23}}\left\{1-\frac{1}{2} \epsilon_{13}-\frac{1}{4}\left(\epsilon_{12}+\epsilon_{23}\right)\right\} \\
g_{23}^{*}=\sqrt{\epsilon_{12} \epsilon_{13}}\left\{1-\frac{1}{2} \epsilon_{23}-\frac{1}{4}\left(\epsilon_{12}+\epsilon_{13}\right)\right\}
\end{array}\right.
$$

They correspond to the non-trivial fixed point of the coupled models which we are interested in. It is readily checked that the fixed point (2.13) is stable in one direction and unstable in the two others, as is the case when one couples identical models. Furthermore, the topology of the RG flows is similar.

Substituting the fixed point values of the couplings in the matrix $\Lambda_{a b}$ and diagonalising it, one obtains, after some algebra, the following expressions for the eigenvalues:

$$
\begin{aligned}
\lambda_{1} & =-\frac{a+b+c}{2}+\frac{3 a b c}{a+b+c} \\
\lambda_{2,3} & =\frac{a+b+c}{2}-\frac{6 a b c+a^{2} b+a b^{2}+a^{2} c+a c^{2}+b^{2} c+b c^{2}}{2(a+b+c)} \\
& \pm \frac{1}{2(a+b+c)}\left\{6 a^{2} b^{2} c^{2}-2 a b c\left(a^{2} b+a b^{2}+a^{2} c+a c^{2}+b^{2} c+b c^{2}+a^{3}+b^{3}+c^{3}\right)\right. \\
& \left.+a^{2} b^{2}(a+b)^{2}+a^{2} c^{2}(a+c)^{2}+b^{2} c^{2}(b+c)^{2}\right\}^{1 / 2}
\end{aligned}
$$

where we have simplified the notation by means of the abbreviations $a=\epsilon_{12}, b=\epsilon_{13}$ and $c=\epsilon_{23}$. According to the definition (2.11) of $\Lambda_{a b}$, the dimensions of the new primary operators $\left\{\varepsilon_{a}^{*}\right\}$, cf. Eq. (1.5), are related to the above eigenvalues through

$$
\Delta\left(\varepsilon_{a}^{*}\right)=1-\lambda_{a}
$$

for $a=1,2,3$. We recall that all these calculations have been done to second order in $\epsilon$.

It should be remarked that in the special case of two of the parameters being equal, $b=c$ and $a \neq b$, the expressions for the dimensions simplify considerably. One finds:

$$
\begin{aligned}
\Delta\left(\varepsilon_{1}^{*}\right) & =1+\frac{a+2 b}{2}-\frac{3 a b^{2}}{a+2 b} \\
\Delta\left(\varepsilon_{2}^{*}, \varepsilon_{3}^{*}\right) & =1-\frac{a+2 b}{2}+\frac{b\left(4 a b+a^{2}+b^{2}\right)}{a+2 b} \pm \frac{b^{2}(a-b)}{a+2 b}
\end{aligned}
$$


At first order in $\epsilon$, the $\mathrm{RG}$ results for the dimensions

$$
\begin{aligned}
\Delta\left(\varepsilon_{1}^{*}\right) & =1+\frac{a+b+c}{2} \\
\Delta\left(\varepsilon_{2}^{*}\right)=\Delta\left(\varepsilon_{3}^{*}\right) & =1-\frac{a+b+c}{2}
\end{aligned}
$$

form a singlet and a doublet, in accordance with the scenario for the restoration of the $S_{3}$ symmetry discussed in the Introduction. The degeneracy of the doublet is however lifted at order $\epsilon^{2}$, by the last term in (2.15). This is true even when two of the models are identical, and only for $a=b=c$ does one recover the degeneracy $\Delta\left(\varepsilon_{2}^{*}\right)=\Delta\left(\varepsilon_{3}^{*}\right)$ [2].

In section 0, where the results of our numerical work are presented, we shall show that this last result of the perturbative $R G$ is wrong. We provide evidence that the splitting between the dimensions of $\varepsilon_{2}^{*}$ and $\varepsilon_{3}^{*}$ is actually zero, and that the symmetry restoration scenario therefore holds true.

We could suggest the following argument for the failure of the perturbative RG. The $\epsilon$-expansion calculations are valid for perturbed conformal theories because they respect the conformal symmetry, just as dimensional regularisation is valid in the context of pertubative calculations in a gauge theory because the method respects the gauge symmetry (otherwise the results would be dependent on the regularisation technique). In the present problem, the $\epsilon$-regularisation should be correct as far as the conformal symmetry alone is concerned. But in case of extra symmetries, such as $S_{3}$, the method might well give wrong results.

The $R G$ formulae (2.14)-(2.16) for the scaling dimensions, and for the central charge which will be given below, are still quite useful. Apart from the degeneracy issue just discussed, they compare well with the numerical results of section 5 when $\left\{\epsilon_{a}\right\}$ are small enough.

As far as the dimensions $\Delta\left(\varepsilon_{2}^{*}\right), \Delta\left(\varepsilon_{3}^{*}\right)$, defined by Eqs. (2.15)-(2.16), are concerned, it is their mean value which compares well with numerical results. It is worth noticing that the splitting, namely the third term in (2.15), is numerically smaller than the principal

\footnotetext{
${ }^{5}$ We would still claim, of course, that our RG calculations are technically correct!
} 
$\epsilon^{2}$ term, the second term in (2.15). This is because of the compensation of negative and positive terms in the third term of (2.15). . $^{\circ}$

The central charge can be obtained in a simple way using Zamolodchikov's $c$-theorem 88. This theorem provides us with a function of the couplings $c\left(g_{12}, g_{13}, g_{23}\right)$ which decreases along the renormalisation flow and takes a value $c\left(g_{12}^{*}, g_{13}^{*}, g_{23}^{*}\right)$ at the fixed point of the flow which equals the central charge of the associated conformal field theory.

With the conventions (2.5) taken into account, the $c$-function is uniquely determined by

$$
\begin{aligned}
\frac{\partial c\left(g_{12}, g_{13}, g_{23}\right)}{\partial g_{a b}} & =-\frac{3}{2} \beta_{a b}\left(g_{12}, g_{13}, g_{23}\right) \\
c(0,0,0) & =c_{0} \equiv c_{1}+c_{2}+c_{3}
\end{aligned}
$$

where $c_{0}$ is the total central charge of three decoupled models.

From Eq. 2.6) and Eqs. (2.21)-(2.22) the $c$-function turns out to be given by:

$$
c\left(g_{12}, g_{13}, g_{23}\right)=c_{0}-\frac{3}{4}\left(a g_{12}^{2}+b g_{13}^{2}+c g_{23}^{2}\right)+\frac{3}{2} g_{12} g_{13} g_{23}+\frac{3}{8}\left(g_{12}^{2} g_{13}^{2}+g_{12}^{2} g_{23}^{2}+g_{13}^{2} g_{23}^{2}\right)
$$

At the fixed point we insert Eq. (2.13) into Eq. (2.23); up to the order $\epsilon^{4}$ the correction $\Delta c$ of the central charge is:

$$
\Delta c=-\frac{3}{4} a b c+\frac{3}{8}\left(a b c^{2}+b c a^{2}+c a b^{2}\right)
$$

\section{Duality transformations}

The possibility of endowing the Potts model with a duality transformation was one of the main motivations for introducing it [9]. The study of duality for several Potts models coupled by their local energy density was initiated by Domany and Riedel, who worked out the case of two models with $q_{1}$ and $q_{2}$ states [10]. Technically, these authors used a particular version of the method of lattice Fourier transforms [11, due to $\mathrm{Wu}$ and

\footnotetext{
${ }^{6}$ It is easy to check that the expression under the square-root sign of (2.15) is always non-negative, so that $\lambda_{2,3}$ are well defined.
} 
Wang [12]. Dotsenko et al. generalised the computations to three symmetrically coupled $q$-state models [2]. However, it became clear that the complexity of the Fourier method grew rapidly as the number of models to be coupled was increased. Recently, it was pointed out by Jacobsen that trading the Potts spin variables for a formulation in terms of random clusters [13], or loops [14], the duality relations simplified dramatically. This observation made it possible to work out the case of $M$ coupled $q$-state Potts models, with the most general coupling by local energy densities consistent with an $S_{M}$ symmetry [6.

We now show how to treat the even more general case, where each model does not necessarily have the same number of states, and the coupling by local energy densities is the most general one.

\subsection{General case}

Consider a set $\mathcal{L}^{M}$ of $M$ identical planar lattices $\mathcal{L}$, which we imagine to be stacked on top of one another. On each lattice site $i \in \mathcal{L}$, and for each layer $\mu=1,2, \ldots, M$, we define a Potts spin $\sigma_{i}^{(\mu)}$ taking the values $\sigma_{i}^{(\mu)}=1,2,3, \ldots, q_{\mu}$. The layers interact by means of the reduced Hamiltonian

$$
\mathcal{H}=\sum_{\langle i j\rangle} \mathcal{H}_{i j}
$$

where $\langle i j\rangle$ denotes the set of lattice edges, and the nearest-neighbour interaction is defined as

$$
\mathcal{H}_{i j}=-\sum_{\ell \subset \mathcal{E}} K_{\ell} \prod_{\mu \in \ell} \delta\left(\sigma_{i}^{(\mu)}, \sigma_{j}^{(\mu)}\right)
$$

By definition, the Kronecker delta function $\delta(x, y)=1$ if $x=y$, and zero otherwise. We have here defined $\mathcal{E}$ as the product set $\prod_{\mu=1}^{M}\left\{\emptyset, \mathcal{L}^{(\mu)}\right\}$, so any one of the $2^{M}$ subsets in $\mathcal{E}$ can be interpreted as a certain subset of layer indices. One can also think of $\ell \subset \mathcal{E}$ as specifying the state of a given edge $\langle i j\rangle$, meaning that in the interaction term (3.2), $\ell$ determines which of the layers contribute to the product $\prod_{\mu \in \ell}$ of the corresponding delta functions. The layers specified by some $\ell \subset \mathcal{E}$ then interact by means of the product of their local energy densities, through a coupling constant $K_{\ell}$. 
For later convenience, we shall represent a subset $\ell \subset \mathcal{E}$ as a list of $M$ open (o) or filled $(\bullet)$ circles, the $\mu$ th circle indicating respectively the absence (or presense) of the factor $\delta\left(\sigma_{i}^{(\mu)}, \sigma_{j}^{(\mu)}\right)$. Furthermore, we sometimes use a single open (resp. filled) circle as an abbreviation of a list of $M$ open (resp. filled) circles.

When $M=1$, the model defined by Eq. (3.2) reduces to the conventional Potts model, whilst for $M=2$ it is identical to the Ashkin-Teller like model considered in Ref. [10]. For $M=3$, it is the asymmetric version of the model discussed in Ref. [2], which had $q \equiv q_{1}=q_{2}=q_{3}$, and whose couplings possessed an $S_{3}$ symmetry upon permutation of the layers. Using the above symbolic notation, this symmetry can be expressed by the identities

$$
\begin{aligned}
& K_{1} \equiv K_{\bullet \bullet \circ}=K_{\bullet \bullet}=K_{\circ \bullet \bullet} \\
& K_{2} \equiv K_{\bullet \bullet \bullet}=K_{\bullet \bullet}=K_{\bullet \bullet \bullet} \\
& K_{3} \equiv K_{\bullet \bullet \bullet}
\end{aligned}
$$

By means of a generalised Kasteleyn-Fortuin transformation [13] the local Boltzmann weights can be recast as

$$
\exp \left(-\mathcal{H}_{i j}\right)=\prod_{\ell \subset \mathcal{E}}\left[1+\left(\mathrm{e}^{K_{\ell}}-1\right) \prod_{\mu \in \ell} \delta\left(\sigma_{i}^{(\mu)}, \sigma_{j}^{(\mu)}\right)\right],
$$

where we have simply used that $\exp (K \delta)=1+[\exp (K)-1] \delta$, if $\delta$ can only take the values 0 and 1 . Since furthermore $\delta^{2}=\delta$, expanding the product over $\ell$ will lead to the equivalent form

$$
\exp \left(-\mathcal{H}_{i j}\right)=b_{\circ}+\sum_{\ell \subset \mathcal{E}} b_{\ell} \prod_{\mu \in \ell} \delta\left(\sigma_{i}^{(\mu)}, \sigma_{j}^{(\mu)}\right),
$$

defining the coefficients $b_{\ell}$. The normalisation of Eq. (3.4) is expressed by the fact that $b_{\circ}=1$.

To relate the $b_{\ell}$ to the physical coupling constants $K_{\ell}$, we evaluate Eqs. (3.4) and (3.5) in the situation where $\delta\left(\sigma_{i}^{(\mu)}, \sigma_{j}^{(\mu)}\right)=1$ for $\mu \in \ell^{\prime}$, and zero otherwise. This resulting equations

$$
\exp \left(\sum_{\rho \subset \ell^{\prime}} K_{\rho}\right)=\sum_{\rho \subset \ell^{\prime}} b_{\rho}
$$


can readily be solved by applying the principle of inclusion-exclusion [15], yielding

$$
b_{\ell}=\sum_{\ell^{\prime} \subset \ell}(-1)^{|\ell|-\mid \ell^{\prime \mid}} \exp \left(\sum_{\rho \in \ell^{\prime}} K_{\rho}\right) .
$$

The partition function in the spin representation

$$
Z=\sum_{\{\sigma\}} \prod_{\langle i j\rangle} \exp \left(-\mathcal{H}_{i j}\right)
$$

can now be transformed into the random cluster representation as follows. First, insert Eq. (3.5) on the right-hand side of the above equation, and imagine expanding the product over the lattice edges $\langle i j\rangle$. To each term in the resulting sum we associate an edge colouring $\mathcal{G}$ of $\mathcal{L}^{M}$, where an edge $\langle i j\rangle$ in layer $\mu$ is considered to be coloured (occupied) if the term contains the factor $\delta\left(\sigma_{i}^{(\mu)}, \sigma_{j}^{(\mu)}\right)$, and uncoloured (empty) if it does not. In other words, an edge colouring $\mathcal{G}$ is determined by specifying an edge state $\ell_{i j} \subset \mathcal{E}$ for every edge $\langle i j\rangle \in \mathcal{L}$.

The summation over the spin variables $\{\sigma\}$ is now trivially performed, yielding a factor of $q_{\mu}$ for each connected component (cluster) in layer $\mu$ of the colouring graph. Keeping track of the prefactors multiplying the $\delta$-functions, using Eq. (3.5), we conclude that

$$
Z=\sum_{\mathcal{G}} \prod_{\ell \subset \mathcal{E}} b_{\ell}^{B_{\ell}} \prod_{\mu \in \ell} q_{\mu}^{C_{\mu}}
$$

where $C_{\mu}$ is the number of clusters in layer $\mu$, and $B_{\ell}$ is the number of edges $\langle i j\rangle \in \mathcal{L}^{M}$ having the state $\ell$.

It is worth noticing that the random cluster description of the model has the advantage that the $q_{\mu}$ only enter as parameters. By analytic continuation one can thus give meaning to a non-integer number of states. The price to be paid is that the $C_{\mu}$ are, a priori, nonlocal quantities.

In terms of the edge variables $b_{\ell}$ the duality transformation of the partition function is easily worked out. For simplicity we shall assume that the couplings constants $K_{\ell}$ are identical between all nearest-neighbour pairs of spins. The generalisation to an arbitrary inhomogeneous distribution of couplings is trivial; it suffices to let $K_{\ell}$ depend on $\langle i j\rangle$ in Eq. (3.4). 
By definition, we take the colouring state $\ell \subset \mathcal{E}$ of the edge $\langle i j\rangle \in \mathcal{L}$ to be dual to the complementary colouring $\ell^{*}$ of its intersecting dual edge $\widetilde{\langle i j\rangle} \in \tilde{\mathcal{L}}$. In the symbolic notation introduced above, the complementarity operation $*$ simply means replacing every $\bullet$ by a $\circ$, and vice versa. Also, note that we refer to dual quantities by a tilde throughout.

To establish the duality transformations, we begin by postulating that the configuration $\mathcal{G}$ • with all lattice edges coloured, be dual to the configuration $\mathcal{G}_{\bullet}^{*} \equiv \mathcal{G}_{\circ}$ with no coloured (dual) edge.

This requirement fixes the constant entering the duality transformation. Indeed, from Eq. (3.9), we find that $\mathcal{G}_{\bullet}$ has weight $b_{\bullet}^{E} \prod_{\mu=1}^{M} q_{\mu}$, where $E$ is the total number of lattice edges, and $\mathcal{G}_{\circ}$ is weighted by $\tilde{b}_{\circ}^{E} \prod_{\mu=1}^{M} q_{\mu}^{F}$, where $F$ is the number of faces, including the exterior one. We thus seek for a duality transformation of the form

$$
\frac{Z\left(\left\{b_{\ell}\right\}\right)}{\tilde{Z}\left(\left\{\tilde{b}_{\ell}\right\}\right)}=\left(\tilde{b}_{\circ} / b_{\bullet}\right)^{E} \prod_{\mu=1}^{M} q_{\mu}^{F-1},
$$

where for any configuration $\mathcal{G}$ the edge weights must transform so as to keep the same relative weight between $\mathcal{G}$ and $\mathcal{G}_{\bullet}$ as between $\mathcal{G}^{*}$ and $\mathcal{G}_{\text {。 }}$.

An arbitrary colouring configuration $\mathcal{G}$ entering Eq. (3.9) can be generated by applying a finite number of changes to $\mathcal{G}_{\bullet}$, in which an edge of weight $b_{\bullet}$ is changed into an edge of weight $b_{\ell}$ for some $\ell \subset \mathcal{E}$. By such a change, in general, a pivotal bond is removed from the colouring graph in each of some subset $\ell^{\prime} \subset \ell^{*}$ of layers, thus creating $\left|\ell^{\prime}\right|$ new clusters in the corresponding layers. The weight relative to that of $\mathcal{G}$. will therefore change by $\left(b_{\ell} / b_{\bullet}\right) \prod_{\mu \in \ell^{\prime}} q_{\mu}$. On the other hand, in the dual configuration $\tilde{\mathcal{G}}$ a cluster will be lost in each of the layers $\left(\ell^{\prime}\right)^{*} \cap \ell^{*}$, since each of the $\left|\ell^{\prime}\right|$ new clusters mentioned above will be accompanied by the formation of a loop in $\tilde{\mathcal{G}}$. The weight change relative to $\mathcal{G}_{\circ}$ therefore amounts to $\left(\tilde{b}_{\ell^{*}} / \tilde{b}_{0}\right) \prod_{\mu \in\left(\ell^{\prime}\right)^{*} \cap \ell^{*}} q_{\mu}^{-1}$. Comparing these two changes we see that the factor $\prod_{\mu \in \ell^{\prime}} q_{\mu}$ cancels nicely, and the duality transformation takes the simple form

$$
\tilde{b}_{\ell}=\left(b_{\ell^{*}} / b_{\bullet}\right) \prod_{\mu \in \ell} q_{\mu},
$$

the relation with $\ell=\circ$ being trivial. 
Note in particular that Eq. (3.11) with $\ell=\bullet$ implies that $b_{\bullet} \tilde{b}_{\bullet}=\prod_{\mu=1}^{M} q_{\mu}$, since $b_{\circ}=1$ by definition. Then, dualising Eq. (3.11) once again yields

$$
\widetilde{\widetilde{b}}_{\ell}=\frac{1}{\tilde{b}} \prod_{\bullet \in \ell} q_{\mu} \cdot \frac{b_{\ell}}{b_{\bullet}} \prod_{\mu \in \ell^{*}} q_{\mu}=b_{\ell},
$$

so that the duality transformation is indeed involutive, as required.

The duality relations (3.11) can be recast in an even simpler form by trading the random clusters for the loops surrounding them (and their duals) on the lattice $\mathcal{L}_{\mathrm{m}}$ medial to $\mathcal{L}$ [14. Using the Euler relation, we find that Eq. (3.9) must be replaced by

$$
Z=\left(\prod_{\mu=1}^{M} q_{\mu}\right)^{N / 2} \sum_{\mathcal{G}} \prod_{\ell \subset \mathcal{E}} x_{\ell}^{B_{\ell}} \prod_{\mu \in \ell} q_{\mu}^{L_{\mu} / 2}
$$

where, by a slight abuse of notation, we use the same notation $\mathcal{G}$ for the loop and the cluster configurations, since they are in bijective correspondence. $L_{\mu}$ are now the number of closed loops in layer $\mu$, and $N$ is the total number of vertices in $\mathcal{L}$. Note that the bond weights $b_{\ell}$ have now been replaced by

$$
x_{\ell}=b_{\ell} \prod_{\mu \in \ell} q_{\mu}^{-1 / 2}
$$

It is easily verified that the duality relations (3.11) now simply read

$$
\tilde{x}_{\ell}=x_{\ell^{*}}
$$

This is our main result.

In the case of a lattice $\mathcal{L}=\tilde{\mathcal{L}}$ which is unchanged by the duality, such as the infinite square lattice, we can now search for selfdual solutions. These are obtained by imposing $\tilde{b}_{\ell}=b_{\ell}$, and read explicitly

$$
x_{\ell}^{(\text {s.d. })}=x_{\ell^{*}}^{(\text {s.d. })}
$$

Since $x_{\circ}=1$ by normalisation, the selfdual manifold has dimension $D_{M}=2^{M-1}-1$.

Two special points always belong to the selfdual manifold. The first one is

$$
x_{\ell}=1, \quad \text { for any } \ell \subset \mathcal{E} \text {. }
$$


It is straightforward to verify that in terms of the original couplings this means $K_{0 \ldots \circ \bullet \cdots \circ}=$ $1+q_{\mu}^{1 / 2}$ (with the unique $\bullet$ at position $\mu$ ) for $\mu=1,2, \ldots, M$, all other $K_{\ell}$ being zero. In other words, this is just $M$ non-interacting selfdual (critical) Potts models.

The other special point is

$$
x_{\circ}=x_{\bullet}=1, \quad\left(\text { all other } x_{\ell}=0\right) .
$$

In terms of the original couplings this means $K_{\bullet}=1+\prod_{\mu=1}^{M} q_{\mu}^{1 / 2}$, all other $K_{\ell}$ being zero. In this case, the $M$ models effectively couple so as to form a single critical Potts model with $\prod_{\mu=1}^{M} q_{\mu}$ states.

\subsection{Two models}

Let us briefly show how to recover the result for $M=2[10]$ from the compact formulation of Eq. (3.15).

Introducing the shorthand notation $\delta_{\mu}=\delta\left(\sigma_{i}^{(\mu)}, \sigma_{j}^{(\mu)}\right)$ for $\mu=1,2$, the Hamiltonian (3.2) reads in this case

$$
-\mathcal{H}_{i j}=K_{\bullet} \delta_{1}+K_{\bullet \bullet} \delta_{2}+K_{\bullet \bullet} \delta_{1} \delta_{2}
$$

From Eq. (3.7) we have

$$
\begin{aligned}
\sqrt{q_{1}} x_{\bullet \bullet} & \equiv b_{\bullet \bullet}=\mathrm{e}^{K_{\bullet \bullet}}-1 \\
\sqrt{q_{2}} x_{\bullet \bullet} & \equiv b_{\bullet \bullet}=\mathrm{e}^{K_{\bullet} \bullet}-1 \\
\sqrt{q_{1} q_{2}} x_{\bullet \bullet} & \equiv b_{\bullet \bullet}=\mathrm{e}^{K_{\bullet \bullet}+K_{\bullet}+K_{\bullet} \bullet}-\left(\mathrm{e}^{K_{\bullet}}+\mathrm{e}^{K_{\bullet \bullet}}\right)+1,
\end{aligned}
$$

and using the selfduality criteria $x_{\bullet \bullet}=x_{\bullet \bullet}$ and $x_{\bullet \bullet}=x_{\circ \circ} \equiv 1$ we readily find the solutions

$$
\begin{aligned}
\mathrm{e}^{K_{\bullet}} & =1+\sqrt{\frac{q_{2}}{q_{1}}}\left(\mathrm{e}^{K_{\bullet}}-1\right) \\
\mathrm{e}^{K} \bullet & =\frac{\sqrt{q_{1}}+\sqrt{q_{2}}\left[1+\left(q_{1}-1\right) \mathrm{e}^{-K_{\bullet}}\right]}{\sqrt{q_{1}}+\sqrt{q_{2}}\left(\mathrm{e}^{K_{\bullet}}-1\right)} .
\end{aligned}
$$




\subsection{Three models}

For the case $M=3$, the Hamiltonian (3.2) reads

$$
\begin{aligned}
-\mathcal{H}_{i j}= & K_{\bullet \circ} \delta_{1}+K_{\circ \bullet \circ} \delta_{2}+K_{\circ \bullet \bullet} \delta_{3}+ \\
& K_{\bullet \bullet \bullet} \delta_{2} \delta_{3}+K_{\bullet \bullet \bullet} \delta_{1} \delta_{3}+K_{\bullet \bullet \circ} \delta_{1} \delta_{2}+K_{\bullet \bullet \bullet} \delta_{1} \delta_{2} \delta_{3} .
\end{aligned}
$$

From Eq. (3.7) we have

$$
\begin{aligned}
& b_{\bullet \circ \circ}=\mathrm{e}^{K \bullet \bullet \circ}-1 \\
& b_{\circ \bullet \bullet}=\mathrm{e}^{K_{\bullet \bullet \bullet+K}+K_{\bullet \bullet}+K_{\circ \circ}}-\left(\mathrm{e}^{K_{\circ \bullet \bullet}}+\mathrm{e}^{K_{\circ \circ \bullet}}\right)+1
\end{aligned}
$$

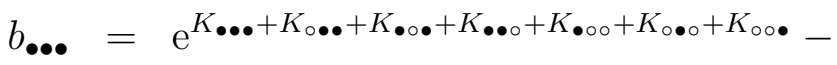

$$
\begin{aligned}
& \left(\mathrm{e}^{K_{\circ} \bullet \bullet+K_{\bullet \bullet}+K_{\circ} \bullet}+\mathrm{e}^{K \bullet \bullet \bullet+K \bullet \circ \circ+K_{\circ} \bullet}+\mathrm{e}^{K \bullet \bullet \circ+K \bullet \bullet \circ+K_{\circ} \bullet}\right)+ \\
& \left(\mathrm{e}^{K \bullet \bullet \circ}+\mathrm{e}^{K_{\circ \bullet \bullet}}+\mathrm{e}^{K_{\circ} \bullet}\right)-1,
\end{aligned}
$$

where the first and second line each represent three equations that can be obtained by cyclically rotating the layer indices.

Imposing the selfduality criteria $\sqrt{q_{1}} b_{\bullet \bullet \bullet}=\sqrt{q_{2} q_{3}} b_{\bullet \circ \circ}$ and $b_{\bullet \bullet \bullet}=\sqrt{q_{1} q_{2} q_{3}}$ we obtain after a little algebra

$$
\begin{aligned}
& \mathrm{e}^{K_{\circ} \bullet}=\frac{\sqrt{\frac{q_{2} q_{3}}{q_{1}}}\left(\mathrm{e}^{K \bullet \bullet \circ}-1\right)+\mathrm{e}^{K_{\circ} \bullet}+\mathrm{e}^{K_{\circ \circ \bullet}}-1}{\mathrm{e}^{K_{\circ} \bullet+K_{\circ} \bullet}} \\
& \mathrm{e}^{K \bullet \bullet \bullet}=\frac{A}{B} \cdot \mathrm{e}^{K \bullet \bullet \circ+K_{\circ} \bullet+K_{\circ} \bullet} \\
& A=\left(\mathrm{e}^{K \bullet \bullet \circ}-1\right) q_{2} q_{3}+\left(\mathrm{e}^{K_{\circ} \bullet}-1\right) q_{1} q_{3}+\left(\mathrm{e}^{K_{\circ \circ}}-1\right) q_{1} q_{2}+ \\
& \left(\mathrm{e}^{K \bullet \bullet \circ}+\mathrm{e}^{K_{\circ} \bullet}+\mathrm{e}^{K_{\circ \circ}}-2\right) \sqrt{q_{1} q_{2} q_{3}}+q_{1} q_{2} q_{3} \\
& B=\left[\left(\mathrm{e}^{K_{\bullet \bullet}}+\mathrm{e}^{K_{\circ} \bullet}-1\right) \sqrt{q_{1}}+\left(\mathrm{e}^{K \bullet \bullet \circ}-1\right) \sqrt{q_{2} q_{3}}\right] \times \\
& {\left[\left(\mathrm{e}^{K \bullet \bullet \circ}+\mathrm{e}^{K_{\circ} \bullet}-1\right) \sqrt{q_{2}}+\left(\mathrm{e}^{K_{\circ} \bullet}-1\right) \sqrt{q_{1} q_{3}}\right] \times} \\
& {\left[\left(\mathrm{e}^{K \bullet \bullet \circ}+\mathrm{e}^{K_{\circ} \bullet}-1\right) \sqrt{q_{3}}+\left(\mathrm{e}^{K_{\circ} \bullet}-1\right) \sqrt{q_{1} q_{2}}\right] .}
\end{aligned}
$$

These expressions generalise those given in Ref. [2]. 
It should be clear that for higher $M$, manipulating such expressions by direct use of the Fourier method [12] becomes extremely cumbersome.

\section{Transfer matrix algorithm}

In Ref. [2] it was shown that the transfer matrix $T(L)$ for coupled Potts models on semiinfinite strips of width $L$ may be written in a variety of ways. For a given $L$, the smaller the dimension of $T(L)$ the more efficient will be the computations, since both time and memory consumption increase roughly linearly with $\operatorname{dim} T(L)$.

The best choice turns out to be to write the transfer matrix for the loop model on the medial lattice $\mathcal{L}_{\mathrm{m}}$ [14, which was referred to as algorithm alg4 in [2]. This also gives us the advantage of having simple duality relations, cf. Eq. (3.15), and to be able to treat the numbers of states $\left\{q_{1}, q_{2}, q_{3}\right\}$ as continuous parameters.

Let us recall from Eqs. (3.13) and (3.16) that the partition function for $M=3$ coupled models on the selfdual manifold can be written, up to a trivial multiplicative constant, as

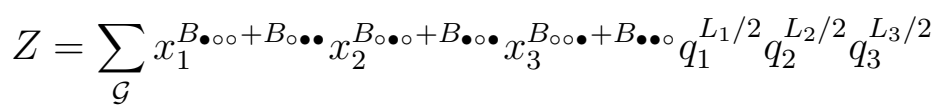

where we have put $x_{1}=x_{\bullet \circ \circ}, x_{2}=x_{\circ \bullet \bullet}$ and $x_{3}=x_{\circ \bullet \bullet}$ for brevity. For simplicity, we shall take the lattice $\mathcal{L}$ to be the square one, so that $\mathcal{L}_{\mathrm{m}}$ is once again a square lattice.

In the loop picture, the symbols $\bullet$ and $\circ$ refer to the two different ways of splitting the vertex, with a definition that alternates between the even and the odd sublattice; see Fig. 田. But thanks to the selfduality, Eq. (4.1) is invariant with respect to a global colour conjugation $\bullet \leftrightarrow \circ$, and so one might as well forget about the distinction between the sublattices? The number of occurrences of a given vertex splitting (and hence the $B$ 's) can be counted locally, and are thus easily realised as local Boltzmann factors in the transfer matrix.

\footnotetext{
${ }^{7}$ Using the language of Ref. 14, coupled Potts models constitute a staggered vertex model, which however becomes homogeneous exactly on the selfdual manifold.
} 


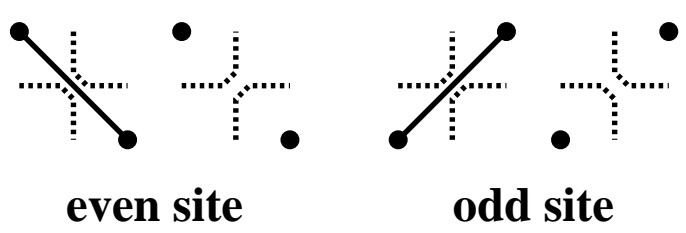

Figure 1: The relation between the random cluster model on the square lattice and the loop model on the corresponding medial graph. The clusters consist of connected components of coloured edges (thick lines) or isolated sites (filled circles). Loops on the medial graph (dashed lines) are defined by the convention that they wrap around the cluster boundaries.

The $L_{\mu}$ in (4.1) count the number of closed loops in each layer $\mu=1,2,3$. Despite of the non-local nature of the loops, these quantities do not obviate the construction of the transfer matrix. Rather, they can be counted locally by writing the transfer matrix in the basis of Catalan-like connectivities, as described in [2]. Formally, if $\mathcal{C}_{k}$ designates the space of pairwise Catalan connectivities among a set of $L=2 k$ points, $T(L)$ acts on the product space $\mathcal{S}_{k}=\mathcal{C}_{k} \otimes \mathcal{C}_{k} \otimes \mathcal{C}_{k}$. One has $\operatorname{dim} \mathcal{C}_{k}=\frac{(2 k) !}{k !(k+1) !}$.

Weighing the loops with a Boltzmann factor $\sqrt{q_{\mu}}$ that depends on the layer $\mu$ is a trivial modification of [2]. An important difference, however, is that the layers are now distinguishable. Accordingly, one has simply $\operatorname{dim} \mathcal{S}_{k}=\left(\operatorname{dim} \mathcal{C}_{k}\right)^{3}$.

As in Ref. [2] we transfer along one of the main directions of the square lattice $\mathcal{L}_{\mathrm{m}}$, with periodic boundary conditions in the transverse direction. To ensure that $\mathcal{C}_{k}$ be well defined, we must take the strip width to be even, $L=2 k$.

We shall also need to consider the situation where all vertex weights $x$ tend to infinity in fixed ratios. Defining $x_{2}^{\prime}=\frac{x_{2}}{x_{1}}$ and $x_{3}^{\prime}=\frac{x_{3}}{x_{1}}$ as the relevant ratios, (4.1) can be rewritten, once again up to an irrelevant multiplicative constant, as

$$
Z^{\prime}=\sum_{\mathcal{G}^{\prime}} x_{2}^{\prime B \circ \bullet \bullet+B \bullet \bullet} x_{3}^{\prime B_{\circ \circ} \bullet+B \bullet \bullet} q_{1}^{L_{1} / 2} q_{2}^{L_{2} / 2} q_{3}^{L_{3} / 2}
$$

where the symbol $\mathcal{G}^{\prime}$ means all colouring figurations in which the local colourings $\circ \circ \circ$ and $\bullet \bullet$ do not occur. In Ref. [2], the special case $\left(x_{2}^{\prime}, x_{3}^{\prime}\right)=(1,1)$ with $q_{1}=q_{2}=q_{3}$ 
was identified as the non-trivial critical fixed point for three identical coupled models.

\section{$5 \quad$ Numerical results}

Using sparse matrix factorisation techniques, we have been able to numerically compute the first few eigenvalues of the transfer matrices $T(L)$ for $Z$ (4.1) and $T^{\prime}(L)$ for $Z^{\prime}(4.2)$ for even strip widths up to $L_{\max }=12$. The largest matrices had dimension $\operatorname{dim} T\left(L_{\max }\right)=$ $(132)^{3}$, but the sparse matrix factorisation necessitates the use of intermediate states with $L+2$ dangling loop segments, and so the largest sparse matrices involved were of dimension $(429)^{3}$.

\subsection{Phase diagram and central charge}

As a first test of our algorithm, we checked that it gave the correct eigenvalues at the special points $\left(x_{1}, x_{2}, x_{3}\right)=(1,1,1)$ and $(0,0,0)$, cf. Eqs. (3.17) and (3.18).

We then extracted the (effective) central charge from the leading eigenvalue $\lambda_{0}$ in the standard way [16, 17]:

$$
f_{0}(L)=f_{0}(\infty)-\frac{\pi c}{6 L^{2}}+\cdots
$$

with $f_{0}(L)=-\frac{1}{L} \log \lambda_{0}$. Using the $c$-theorem [8], we could readily establish the topology of the RG flows:

- In the space $\left(x_{1}, x_{2}, x_{3}\right)$, there is a one-parameter curve along which the partial derivatives of the effective central charge with respect to the two perpendicular directions vanish identically. Moreover, the corresponding second derivatives are strictly negative, so that this curve acts as a "mountain ridge" for $c_{\text {eff }}$.

- The curve passes through the points $(0,0,0)$ and $(1,1,1)$, and then goes to infinity with some fixed ratios $\left(x_{2}^{\prime}, x_{3}^{\prime}\right) \equiv\left(x_{2}^{\prime *}, x_{3}^{* *}\right)$ that depend on $\left(q_{1}, q_{2}, q_{3}\right)$.

- For various values $\left(q_{1}, q_{2}, q_{3}\right) \in[2,4]^{3}$, with at most one $q_{a}=2$, we observe that $c_{\text {eff }}$ is a monotonically decreasing function when going along the curve from the 


\begin{tabular}{l|llll}
$L$ & $x_{2}^{*}$ & $x_{3}^{*}$ & $\left(c_{\mathrm{eff}}\right)_{\max }$ & $\Delta c$ \\
\hline $2,4,6$ & $0.8523(1)$ & $0.7133(1)$ & 1.93540138 & -0.00058 \\
$4,6,8$ & $0.8476(1)$ & $0.7136(1)$ & 1.98040147 & -0.00352 \\
$6,8,10$ & $0.8481(1)$ & $0.7076(1)$ & 1.99047341 & -0.00416 \\
$8,10,12$ & $0.8483(1)$ & $0.7061(1)$ & 1.99348414 & -0.00443
\end{tabular}

Table 1: Maxima of the effective central charge for the system $\mathcal{M}_{345}$, and the deviation from the value at the decoupling point.

point $(1,1,1)$ towards either $(0,0,0)$ or $x_{1}\left(1, x_{2}^{\prime *}, x_{3}^{\prime *}\right), x_{1} \rightarrow \infty$. In the former case, the decrease is rapid and gets more pronounced with increasing system size $L$, signalling the first-order nature $\left(c_{\mathrm{eff}}=0\right)$ of the phase transition in the $\left(q_{1} q_{2} q_{3}\right)$ state Potts model. In the latter case, the decrease is very slight, distinguishing the point $\left(x_{2}^{\prime *}, x_{3}^{\prime *}\right)$ as a candidate for the non-trivial fixed point of three coupled Potts models in the lattice realisation.

Based on this evidence, we switched to the matrix $T^{\prime}(L)$ in order to accurately locate the point $\left(x_{2}^{\prime *}, x_{3}^{\prime *}\right)$ and study its critical properties. Invoking again the $c$-theorem [8], this was done by scanning the space $\left(x_{2}^{\prime}, x_{3}^{\prime}\right)$ for a maximum in $c_{\text {eff }}$ for different system sizes. As usually [2], we obtained the best precision by including a non-universal $1 / L^{4}$ term in (5.1), and so our fits are based on three consecutive strip widths $L$. The obtained maximum can be interpreted as a finite-size pseudo-critical point, which tends to $\left(x_{2}^{\prime *}, x_{3}^{\prime *}\right)$ as $L \rightarrow \infty$.

We have concentrated the main part of our computation time on two important special cases: A tricritical Ising model and a three-state Potts model coupled with either an Ising model or with a tricritical three-state Potts model. In the language of minimal models, we refer to these two situations as $\mathcal{M}_{345} \equiv \mathcal{M}_{3} \times \mathcal{M}_{4} \times \mathcal{M}_{5}$ and $\mathcal{M}_{456} \equiv \mathcal{M}_{4} \times \mathcal{M}_{5} \times \mathcal{M}_{6}$ respectively.

The corresponding finite-size estimates for $\left(x_{2}^{\prime *}, x_{3}^{\prime *}\right)$ and for $\left(c_{\mathrm{eff}}\right)_{\max }$ are given in Tables 1 and 2. Rather than directly extrapolating $\left(c_{\text {eff }}\right)_{\max }$ to the $L \rightarrow \infty$ limit, we consider 


\begin{tabular}{l|llll}
$L$ & $x_{2}^{\prime *}$ & $x_{3}^{\prime *}$ & $\left(c_{\mathrm{eff}}\right)_{\max }$ & $\Delta c$ \\
\hline $2,4,6$ & $0.9230(1)$ & $0.8679(1)$ & 2.27445933 & -0.0115 \\
$4,6,8$ & $0.9218(1)$ & $0.8661(1)$ & 2.32198052 & -0.0185 \\
$6,8,10$ & $0.9213(1)$ & $0.8645(1)$ & 2.33129236 & -0.0190 \\
$8,10,12$ & $0.9213(5)$ & $0.8645(5)$ & 2.333272 & -0.0225
\end{tabular}

Table 2: Maxima of the effective central charge for the system $\mathcal{M}_{456}$, and the deviation from the value at the decoupling point.

instead for any $L$ its deviation $\Delta c(L)$ with respect to the corresponding value found at the point where the three Potts models decouple. Since this deviation is numerically small, it is reasonable to expect that the finite-size corrections to $c$ at $\left(x_{2}^{\prime *}, x_{3}^{\prime *}\right)$ are similar to those at the decoupling point. Tables 1 and 2 confirm that this is indeed the case: the estimates for $\Delta c$ only depend very weakly on $L$. As $L \rightarrow \infty$, we obtain finally the following extrapolated values for $\Delta c$ and for the critical couplings:

$$
\begin{array}{lll}
\text { Model } \mathcal{M}_{345}: & \left(x_{2}^{\prime *}, x_{3}^{* *}\right)=(0.8485(1), 0.7053(5)) & \Delta c=-0.0050(3) \\
\text { Model } \mathcal{M}_{456}: & \left(x_{2}^{\prime *}, x_{3}^{\prime *}\right)=(0.9210(5), 0.862(2)) & \Delta c=-0.025(3)
\end{array}
$$

The values of $\Delta c$ are seen to compare quite favourably with the two-loop result of the perturbative RG (2.24), whose numerical values read respectively

$$
\Delta c\left(\mathcal{M}_{345}\right)_{\mathrm{RG}}=-0.0041 \quad \Delta c\left(\mathcal{M}_{456}\right)_{\mathrm{RG}}=-0.0175
$$

Actually, assuming that the RG series is alternating, the fifth-order term not present in (2.24) would supposedly lead to even better agreement with the numerical results.

\subsection{Higher exponents in the even sector}

Examining the higher eigenvalues of the transfer matrices, one has also access to the scaling dimensions by using the formula [18]

$$
f_{i}(L)-f_{0}(L)=\frac{2 \pi\left(\Delta_{i}\right)_{\mathrm{phys}}}{L^{2}}+\cdots
$$


We here work in the even sector of the transfer matrix, and besides the identity operator (the free energy) we expect to find the various energy-like primaries. In particular we are interested in the primary operators $\varepsilon_{1}^{*}, \varepsilon_{2}^{*}, \varepsilon_{3}^{*}$ discussed in section 2; cf. Eq. (1.5). According to Eqs. (2.14)-(2.16) we have $0<\Delta\left(\varepsilon_{2}^{*}\right), \Delta\left(\varepsilon_{3}^{*}\right)<1$ and $1<\Delta\left(\varepsilon_{1}^{*}\right)<2$ for small enough $\left\{\epsilon_{a}\right\}$, and so we expect the first three gaps of the transfer matrix to be associated with these operators.

As in Ref. [2], the scaling dimensions of the operators have been extracted by adding to Eq. (5.4) a non-universal $1 / L^{4}$ term, and so the fits are based on two consecutive strip widths $L$.

We consider first the case of coupling three identical models.

At the non-trivial fixed point $\left(x_{2}^{\prime}, x_{3}^{\prime}\right)=(1,1)$ the second and third eigenvalues of the transfer matrix are degenerate. These eigenvalues are associated with the operators $\varepsilon_{\mathrm{A}_{1}}, \varepsilon_{\mathrm{A}_{2}}$ of (1.4) which generate the two-dimensional irrep of $S_{3}$, as discussed in the Introduction.

It is important to notice that in the transfer matrix algorithm of [2] the three layers were treated symmetrically and only the operators which are symmetric under permutation of the layer indices were accessible. In that case, the degenerate eigenvalues associated with the energy doublet were not present in the spectrum of the transfer matrix. The fact that we find them here is a strong confirmation of the antisymmetric nature of the corresponding operators.

In Table 3 we report numerical values of the dimension $\Delta\left(\varepsilon_{\mathrm{A}}\right) \equiv \Delta\left(\varepsilon_{\mathrm{A}_{1}}\right)=\Delta\left(\varepsilon_{\mathrm{A}_{2}}\right)$ in the case of three coupled Potts models with $q=3$. The final result $\Delta\left(\varepsilon_{\mathrm{A}}\right)=0.63(3)$ is in reasonable agreement with the $\mathrm{RG}$ value and also agrees well with the Monte Carlo result $\Delta\left(\varepsilon_{\mathrm{A}}\right)=0.63 \pm 0.04$ given in [2]].

The fourth eigenvalue of the transfer matrix was already found in [2] and it corresponds to the symmetric energy operator $\varepsilon_{\mathrm{S}}$ of Eq. (1.3). It is a non-trivial check of our transfer matrices that the corresponding eigenvalue is identical to that of [2].

We now turn our attention to the case of three different coupled models. 


\begin{tabular}{r|l}
$L$ & $\Delta\left(\varepsilon_{\mathrm{A}}\right)$ \\
\hline 4,6 & 0.6768 \\
6,8 & 0.6648 \\
8,10 & 0.6525 \\
$L \rightarrow \infty$ & $0.63(3)$ \\
$\mathrm{RG}$ result & 0.72
\end{tabular}

Table 3: Dimension of the antisymmetric energy operator at $\left(x_{2}^{\prime}, x_{3}^{\prime}\right)=(1,1)$ for three coupled three-state Potts models.

In the space $\left(x_{2}^{\prime}, x_{3}^{\prime}\right)$ of the couplings we have found that for any given system size $L$ there is a unique point $\left(x_{2}^{\prime}, x_{3}^{\prime}\right)_{\mathrm{deg}}$, at which the second and third eigenvalues are degenerate. We have determined the location of this point for the models $\mathcal{M}_{345}$ and $\mathcal{M}_{456}$ with $L=4,6,8,10$ and it is shown on Figs. 23.

In contradistinction to the case of three coupled identical models, the points $\left(x_{2}^{\prime *}, x_{3}^{* *}\right)$ and $\left(x_{2}^{\prime}, x_{3}^{\prime}\right)_{\text {deg }}$ do not coincide for finite systems. It is however evident from Figs. 2 3 that these two points become closer as the system size increases. This suggests that in the thermodynamic limit they actually coincide. If this scenario holds true, we have at the critical point that $\Delta\left(\varepsilon_{3}^{*}\right)=\Delta\left(\varepsilon_{2}^{*}\right)$.

Since the finite-size effects in the sequence of points $\left(x_{2}^{\prime}, x_{3}^{\prime}\right)_{\text {deg }}$ are much stronger than is the case for $\left(x_{2}^{* *}, x_{3}^{* *}\right)$, it is reasonable to measure numerical values of the dimensions at the best available estimate for the latter point. We display the corresponding results for $\Delta\left(\varepsilon_{1}^{*}\right), \Delta\left(\varepsilon_{2}^{*}\right), \Delta\left(\varepsilon_{3}^{*}\right)$ in Tables 4 5, once again for the models $\mathcal{M}_{345}$ and $\mathcal{M}_{456}$ respectively. The numerical results are in quite good agreement with the two-loop RG calculation of section 2. Notice that in the case of $\Delta\left(\varepsilon_{2}^{*}\right), \Delta\left(\varepsilon_{3}^{*}\right)$ we compare with the RG values with the splitting ignored.

In Tables 4 we have given the results for $\Delta\left(\varepsilon_{2}^{*}\right)$ and $\Delta\left(\varepsilon_{3}^{*}\right)$ separately, although we do in fact believe that the two dimensions coincide. This point of view is corroborated by the fact that the difference between the dimensions of $\varepsilon_{2}^{*}$ and $\varepsilon_{3}^{*}$ decreases rapidly with the 


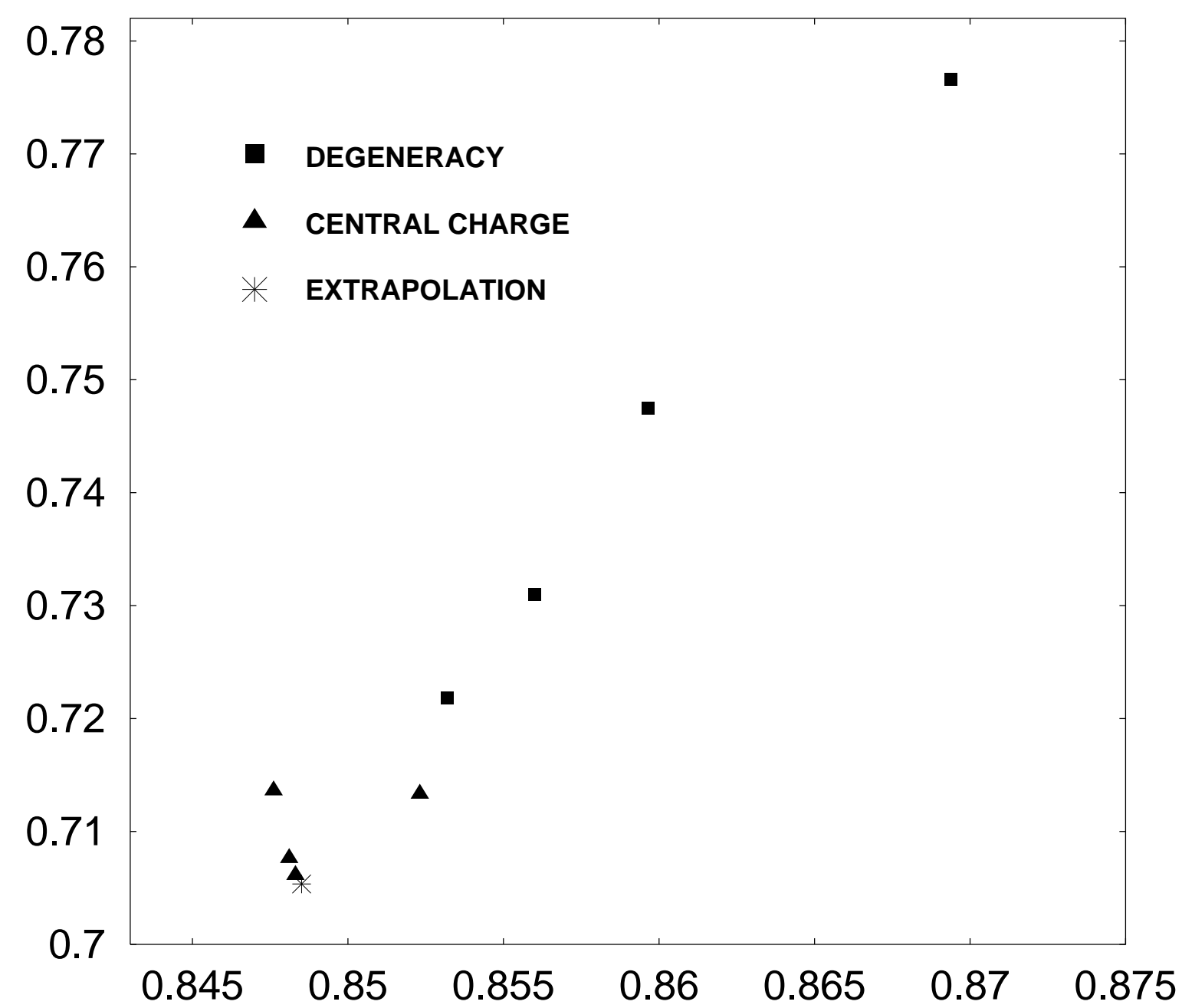

Figure 2: The location of the critical point for the model $\mathcal{M}_{345}$, as determined from the maximum of the effective central charge. Filled triangles show the positions of the maxima $\left(x_{2}^{\prime *}, x_{3}^{\prime *}\right)$ as found from three-point fits with system sizes ranging from $L=2,4,6$ to $L=8,10,12$. The asterix indicates the extrapolation of these points to the thermodynamic limit, $L \rightarrow \infty$. Filled boxes give the positions $\left(x_{2}^{\prime}, x_{3}^{\prime}\right)_{\operatorname{deg}}$ of the degeneracy between the second and third eigenvalues of the transfer matrix, with system sizes ranging from $L=4$ to $L=10$. This latter sequence appears to converge to the same limiting point as the central charge data, in support of the symmetry restoration scenario. 


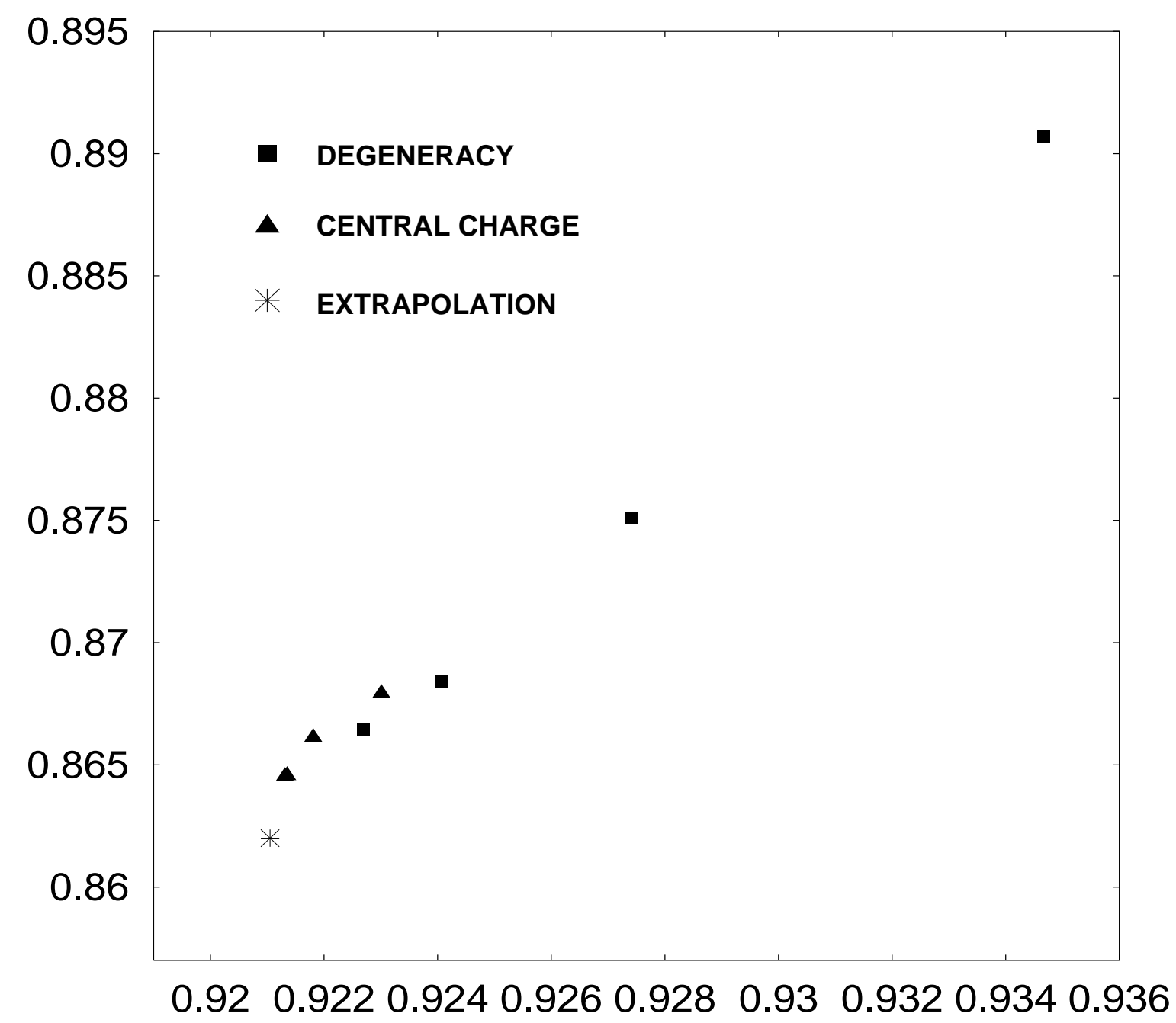

Figure 3: Same data as in Fig. 2, but here for the model $\mathcal{M}_{456}$.

\begin{tabular}{r|lll}
$L$ & $\Delta\left(\varepsilon_{2}^{*}\right)$ & $\Delta\left(\varepsilon_{3}^{*}\right)$ & $\Delta\left(\varepsilon_{1}^{*}\right)$ \\
\hline 4,6 & 0.75734 & 0.7677 & 1.2991 \\
6,8 & 0.759 & 0.763 & 1.2803 \\
8,10 & 0.7564 & 0.75647 & 1.2761 \\
RG result & 0.746 & 0.771 & 1.287
\end{tabular}

Table 4: Dimensions of the energy operators at $\left(x_{2}^{\prime *}, x_{3}^{\prime *}\right)$ for the $\mathcal{M}_{345}$ model. 


\begin{tabular}{r|lll}
$L$ & $\Delta\left(\varepsilon_{2}^{*}\right)$ & $\Delta\left(\varepsilon_{3}^{*}\right)$ & $\Delta\left(\varepsilon_{1}^{*}\right)$ \\
\hline 4,6 & 0.6849 & 0.6857 & 1.2958 \\
6,8 & 0.6731 & 0.6756 & 1.2765 \\
8,10 & 0.6621 & 0.6637 & 1.2745 \\
RG result & 0.701 & 0.728 & 1.43
\end{tabular}

Table 5: Dimensions of the energy operators at $\left(x_{2}^{\prime *}, x_{3}^{\prime *}\right)$ for the $\mathcal{M}_{456}$ model.

lattice size. For $L=8,10$ the numerical results give $\Delta_{\text {splitting }} \equiv\left|\Delta\left(\varepsilon_{3}^{*}\right)-\Delta\left(\varepsilon_{2}^{*}\right)\right|<0.0003$ for the $\mathcal{M}_{345}$ model and $\Delta_{\text {splitting }}<0.002$ for the $\mathcal{M}_{456}$ model.

In order to be sure that the very small values for $\Delta_{\text {splitting }}$ obtained at finite size are not somehow accidental, we have determined an upper bound for $\Delta_{\text {splitting }}$ by directly extrapolating the difference between the second and third eigenvalues of the transfer matrix at the point $\left(x_{2}^{\prime *}, x_{3}^{\prime *}\right)$. The result is shown below together with the extrapolated values for the dimensions of the three energy operators:

$$
\begin{aligned}
& \text { Model } \mathcal{M}_{345}: \quad \Delta\left(\varepsilon_{1}^{*}\right)=1.269 \pm 0.002 \\
& \Delta\left(\varepsilon_{2}^{*}\right), \Delta\left(\varepsilon_{3}^{*}\right)=0.750 \pm 0.005 \quad \Delta_{\text {splitting }}<0.003 \\
& \text { Model } \mathcal{M}_{456}: \quad \Delta\left(\varepsilon_{1}^{*}\right)=1.272 \pm 0.002 \\
& \Delta\left(\varepsilon_{2}^{*}\right), \Delta\left(\varepsilon_{3}^{*}\right)=0.645 \pm 0.003 \quad \Delta_{\text {splitting }}<0.002
\end{aligned}
$$

The difference $\Delta_{\text {splitting }}$ is well below the one predicted by the RG calculation, which reads respectively 0.025 and 0.027 for the models $\mathcal{M}_{345}$ and $\mathcal{M}_{456}$. The numerical work thus provides clear evidence that at the non-trivial fixed point the splitting of the dimensions of $\varepsilon_{3}^{*}$ and $\varepsilon_{2}^{*}$ is actually zero.

\section{Discussion}

In this paper we have shown that coupling $M=3$ different Potts models (with $q_{1}, q_{2}, q_{3}>$ 2 and not too large) one obtains a unique non-trivial critical point. The critical properties of this point, in particular its central charge and the values of various energetic scaling 
dimensions, have been determined quite accurately by a perturbative RG treatment and found to be consistent with large-scale numerical simulations. An exception is however the RG prediction that at two-loop order the degeneracy between the two antisymmetric energy operators should be lifted: this prediction has here been discarded on the basis of numerical evidence.

An extension of our investigation to the case of spin-like operators will be published elsewhere 19 .

This work forms part of a larger project [2], in which we examine the possible universality classes of coupled Potts models, and eventually their relation to the randombond Potts model. In particular, substantial evidence has been accumulated that in the random-bond case replica symmetry is not broken, and one can thus hope to make analytical progress by studying the unitary models that result from coupling a certain number of minimal models.

We believe that the symmetry properties of the coupled models play an essential role. It is in the light of this belief that the present work appears to be interesting: even in the absense of an explicit $S_{M}$ symmetry in the initial action, this symmetry appears to be restored at the non-trivial critical point. As far as a putative CFT classification of $S_{M}$ symmetrical critical points goes, we therefore see that the number of models to be classified is potentially very large. Indeed, assuming the conclusions of the $M=3$ case to carry over to a general number $M$ of coupled models, one may expect a distinct $S_{M}$ symmetric universality class to arise from coupling any different set of $M$ minimal models. If this is true, it would call for a substantial number of new CFTs endowed with extended symmetries. Further research along these lines is currently in progress. 


\section{References}

[1] A. W. W. Ludwig, Nucl. Phys. B 330, 639 (1990).

[2] Vl. S. Dotsenko, J. L. Jacobsen, M.-A. Lewis and M. Picco, Nucl. Phys. B 546, 505 (1999).

[3] Vl. S. Dotsenko, X. S. Nguyen and R. Santachiara, hep-th/0104197, to appear in Nucl. Phys. B.

[4] M.-A. Lewis and P. Simon, Phys. Lett. B 435, 159 (1998).

[5] P. Simon, Nucl. Phys. B 515, 624 (1998).

[6] J. L. Jacobsen, Phys. Rev. E. 62, R1 (2000).

[7] Vl. S. Dotsenko, M. Picco and P. Pujol, Nucl. Phys. B 455, 701 (1995),

[8] A. B. Zamolodchikov, Pis'ma Zh. Eksp. Teor. Fiz. 43, 565 (1986) [English translation in JETP Lett. 43 (1986) 730]; Sov. J. Nucl. Phys. 461090 (1987).

[9] R. B. Potts, Proc. Cambr. Philos. Soc. 48, 106 (1952).

[10] E. Domany and E. K. Riedel, Phys. Rev. B 19, 5817 (1979).

[11] R. Savit, Rev. Mod. Phys. 52, 453 (1980).

[12] F. Y. Wu and Y. K. Wang, J. Math. Phys. 17, 439 (1976).

[13] P. W. Kasteleyn and C. M. Fortuin, J. Phys. Soc. Jpn. (suppl.) 26, 11 (1969).

[14] R. J. Baxter, Exactly solved models in statistical mechanics (Academic Press, New York, 1982).

[15] G. D. Birkhoff, Ann. Math. 14, 42 (1912).

[16] H. Blöte, J. L. Cardy and M. P. Nightingale, Phys. Rev. Lett. 56, 742 (1986).

[17] I. Affleck, Phys. Rev. Lett. 56, 746 (1986).

[18] J. L. Cardy, J. Phys. A 16, L355 (1983).

[19] Vl. S. Dotsenko, J. L. Jacobsen, X. S. Nguyen and R. Santachiara, work in progress. 